\title{
USP7 is associated with greater disease activity in systemic lupus erythematosus via stabilization of the IFN $\alpha$ receptor
}

\author{
YING YU $^{1,2}$, ZHAOLIANG SU $^{1}$, ZHEJIONG WANG ${ }^{2}$ and HUAXI XU ${ }^{1}$ \\ ${ }^{1}$ Institute of Laboratory Medicine, Jiangsu Key Laboratory of Laboratory Medicine, Jiangsu University, \\ Zhenjiang, Jiangsu 210013; ${ }^{2}$ Department of Laboratory Medicine, Chinese Medicine Hospital \\ of Zhejiang, Hangzhou, Zhejiang 310006, P.R. China
}

Received April 20, 2016; Accepted March 24, 2017

DOI: $10.3892 / \mathrm{mmr} .2017 .6819$

\begin{abstract}
An improved understanding of the mechanism of interferon (IFN) $\alpha$ activation in systemic lupus erythematosus (SLE) is likely to aid the identification of effective therapeutic targets. Increasing evidence has indicated that the activity of IFN $\alpha$ is mediated by the interplay of ubiquitylation/deubiquitylation enzyme regulators. The present study identified the deubiquitylation enzyme ubiquitin-specific-processing protease 7 (USP7) as a critical regulator of the human IFN $\alpha-2$ receptor (IFNAR1) protein levels. A co-immunoprecipitation assay was used to demonstrate that USP7 was physically associated with IFNAR1 in vivo. A glutathione S-transferase pull down assay revealed that USP7 interacted with IFNAR1 directly in vitro. Furthermore, USP7 may disassemble IFNAR1 dependent poly-ubiquitin chains and stabilize IFNAR1 in vivo. The activation effects of USP7 on the IFN $\alpha$ pathway were confirmed by reverse transcription-quantitative polymerase chain reaction and western blot analysis. Knockdown of USP7 expression consistently reduced the expression levels of signal transducer and activator of transcription (STAT)-1, STAT-2 and selected IFN-inducible genes, including IFN-induced protein with tetratricopeptide repeats 3 , MX dynamin like GTPase 1 and 2'-5'-oligoadenylate synthetase 1 . The present study demonstrated that USP7 was significantly overexpressed in 210 SLE patients compared with healthy controls. Furthermore, the association between USP7 levels, IFN scores, SLE disease activity index scores and anti-double stranded DNA were analyzed and, as expected, positive correlations were demonstrated, indicating that USP7 may be associated with SLE disease activity through the stabilization of IFNAR1.
\end{abstract}

Correspondence to: Professor Huaxi Xu, Institute of Laboratory Medicine, Jiangsu Key Laboratory of Laboratory Medicine, Jiangsu University, 301 Xuefu Road, Zhejiang, Jiangsu 210013 P.R. China E-mail: huaxiyu8700@163.com

Key words: ubiquitin-specific-processing protease 7, systemic lupus erythematosus, human interferon $\alpha-2$ receptor, deubiquitination

\section{Introduction}

Systemic lupus erythematosus (SLE) is a complex systemic autoimmune disease, characterized as a loss of tolerance to nuclear antigens, the deposition of pathogenic autoantibodies and the formation of immune complexes leading to inflammation in multiple organs $(1,2)$. Dysregulated T cells and their associated mechanisms are important in the pathogenesis of this complicated disease. Epigenetic modifications including DNA methylation, histone tail modifications and microRNAs may additionally serve roles in SLE.

The ubiquitin-proteasome and deubiquitination systems are important cellular mechanisms of protein degradation and stabilization, which may influence gene expression and alter cellular functions without modifying the genomic sequence. Understanding the molecular mechanisms that are involved in the pathophysiology of autoimmune diseases is essential for the introduction of effective, target-directed and accepted therapies $(3,4)$.

One notable feature in SLE is the continuous activation of the type I interferon (IFN) system, shared by the majority of SLE patients (5-7). The vascular damage, endothelial progenitor cell misbalance and induced expression of broad signature gene transcripts that reflect induction are primarily due to the central involvement of IFN $\alpha(8,9)$. The type I interferon receptor complex consisting of the human IFN $\alpha-2$ receptor (IFNAR) 1 and IFNAR2 subunits, and cellular responses to IFN $\alpha$, require adequate expression levels of IFNAR1 (10). It has been reported that IFNAR1 may be ubiquitinated by the Skp1-Cullin1-HOS-Roc1 ubiquitin ligase in vitro (11), however, whether the protein level of IFNAR1 is regulated by the deubiquitination system and whether these deubiquitinating enzymes exhibit a role in the clinical outcomes of SLE patients, remains to be elucidated. The deubiquitinases contain two major groups: the ubiquitin C-terminal hydrolase families and the ubiquitin-specific protease (USP) families. Of the USP families, USP7 is an evolutionarily conserved protease that was first reported in 1997 (12) and was revealed to possess various substrates, including Ci/Gli (13), phosphatase and tensin homolog (14), forkhead box protein O4 (15), histone H2B (16) and tumor protein p53 (17), indicating that USP7 exhibits a role in multiple cellular processes. However, the expression pattern 
and function of USP7 in SLE progression remains to be elucidated.

The present study demonstrated that IFNAR1 acted as a substrate for USP7, and that USP7 functioned to stabilize IFNAR1, which was responsible for greater disease activity in SLE.

\section{Materials and methods}

Patient samples. A total of 210 patients with SLE were recruited from the Chinese Medicine Hospital of Zhejiang (Hangzhou, China) between January 2010 and December 2014; all SLE patients fulfilled the American College of Rheumatology criteria for the disease (18), and gave written informed consent. A further 210 control samples were recruited from the outpatient clinics of the Chinese Medicine Hospital of Zhejiang between January 2010 and December 2014, including those diagnosed as normally healthy or with an unrelated condition. Written informed consent was also obtained from control patients. Patients with other autoimmune diseases including celiac disease, autoimmune hepatitis, sarcoidosis, or autoimmune thyroid disease were excluded from the present study. All procedures were approved by the institutional review board of Jiangsu University. Peripheral blood (5 ml) samples were drawn from healthy donors and SLE patients. The clinical disease activity was measured and assessed according to the SLE disease activity index (DAI) 2000; SLEDAI scores $\geq 10$ were defined as active SLE, while SLEDAI scores $<10$ were defined as stable disease (19).

Samples of peripheral blood mononuclear cells (PBMCs) for isolating $\mathrm{T}$ cells were purified using the Rosette Sep $\mathrm{T}$ cell isolation kit by negative selection (cat no. HY2015; HaoYang Biosciences, Tianjin, China). Serum complement 3 and serum complement 4 , double stranded (ds)DNA and anti-nuclear antibodies (ANA) were measured in blood samples in the Department of Laboratory Medicine using a EUROIMMUN ANA profile kit (cat no. DL 1590) and the Sprinter XL Immunofluorescence system (both from EUROIMMUN AG, Luebeck, Germany), according to the manufacturer's protocol, at the Chinese Medicine Hospital of Zhejiang.

Reagents and antibodies. The short interfering (si)RNA transfection was performed using Lipofectamine RNAiMAX (Invitrogen; Thermo Fisher Scientific, Inc., Waltham, MA, USA) and cells were transfected at $70 \%$ confluence, according to the manufacturer's protocol. The 2 USP7 siRNA oligonucleotide sequences that were used were as follows: siUSP7 1 , 5'-CCGGTGTATCTATTGACTGCCCTTTCTCGAGAAAGG GCAGTCAATAGATACATTTTT-3'; siUSP7 2, 5'-CCG GCCTGGATTTGTGGTTACGTTACTCGAGTAACGTAA CCACAAATCCAGGTTTTT-3'. USP7-targeting siRNA and non-targeting siRNA (5'-CCGGUUCUCCGAACGUCACGU TTTTTTTT-3') were obtained from Sigma-Aldrich (Merck KGaA, Darmstadt, Germany).

Antibodies used were as follows: anti-USP7 antibody (cat no. 4833; 1:1,000; Cell Signaling Technology, Danvers, MA, USA), anti-IFNAR1 (cat no. SAB1406003-50UG; 1:1,000; Sigma-Aldrich; Merck KGaA) and anti- $\beta$-actin (cat no. 612656; 1:2,000; BD Biosciences, Franklin Lakes, NJ,
USA). Horseradish peroxidase-conjugated goat anti-mouse immunoglobulin (Ig)G (cat no. sc-2005; 1:3,000) and goat and anti-rabbit IgG (cat no. sc-2004; 1:3,000) secondary antibodies were obtained from Santa Cruz Biotechnology, Inc. (Dallas, TX, USA).

Cell culture. HEK-293T cells was purchased from American Type Culture Collection (Mannasas, VA, USA) and were cultured in Dulbecco's modified Eagle's medium (Invitrogen; Thermo Fisher Scientific, Inc.) supplemented with $10 \%$ fetal bovine serum (Hyclone; GE Healthcare Life Sciences, Logan, UT, USA), $100 \mathrm{U} / \mathrm{ml}$ of penicillin, and $100 \mathrm{U} / \mathrm{ml}$ of streptomycin (Invitrogen; Thermo Fisher Scientific, Inc.). Cells were maintained at $37^{\circ} \mathrm{C}$ in a humidified $5 \% \mathrm{CO}_{2}$ atmosphere.

$R N A$ isolation and reverse transcription quantitative polymerase chain reaction ( $R T-q P C R)$. Total RNA was isolated using TRIzol ${ }^{\circledR}$ (Invitrogen; Thermo Fisher Scientific, Inc.). cDNA was synthesized from $1 \mu \mathrm{g}$ of total RNA using reverse transcriptase (Invitrogen; Thermo Fisher Scientific, Inc.) and the cDNA $(2 \mu \mathrm{g})$ was amplified using the TransStart Top Green qPCR SuperMix kit (cat no. AQ132-23; TransGen, Beijing, China) on an ABI 7500 Real-Time PCR system (Applied Biosystems; Thermo Fisher Scientific, Inc.). GAPDH was used as an internal normalization control. Thermocycling conditions were as follows: Initial denaturation at $95^{\circ} \mathrm{C}$ for $5 \mathrm{~min}$, followed by 40 cycles of denaturation at $95^{\circ} \mathrm{C}$ for $10 \mathrm{sec}$, and annealing and extension at $60^{\circ} \mathrm{C}$ for $30 \mathrm{sec}$. The primers used were as follows: GAPDH, forward GAGAAGTATGACAAC AGCCTC-3', reverse 5'-ATGGACTGTGGTCATGAGTC-3'; IFNAR1, forward GACTCATTTACACCATTTCGCA-3', reverse 5'-TCAATCCTTTCTTCTACACCTG-3'; and USP7 forward ATTCCTAACATTGCCACCAG-3' and reverse 5'-ATTTACACCATTTGCCATCC-3'. Relative gene expression was calculated according to the $2^{-\Delta \Delta \mathrm{Cq}}$ method (20) and normalized to GAPDH. All experiments were performed at least three times.

Co-immunoprecipitation (co-IP) assay. Cells were lysed using cold lysis buffer (50 mM Tris-Cl, pH 7.4; 1\% NP-40; 150 mM $\mathrm{NaCl} ; 1 \mathrm{mM}$ EDTA; and, $0.5 \%$ sodium deoxycholate) and a protease inhibitor cocktail (BD Biosciences) was used to protect cells from degradation. The supernatants of the lysates were incubated with primary antibodies against USP7 and IFNAR1. A total of $2 \mu \mathrm{g}$ normal rabbit and mouse immunoglobin IgG (cat nos. M8645 and G7402; Sigma-Aldrich; Merck $\mathrm{KGaA}$ ) were used as negative control and Pierce ${ }^{\mathrm{TM}}$ Protein A/G Magnetic Beads (cat no. 88802; Pierce; Thermo Fisher Scientific, Inc.) were added to the immune complexes and incubated for $2 \mathrm{~h}$ at $4^{\circ} \mathrm{C}$. The immune complexes were washed 5 times, subjected to SDS-PAGE and detected by western blot analysis.

Western blot analysis. Protein extracts were lysed with a radioimmunoprecipitation assay buffer (Sigma-Aldrich; Merck KGaA) containing phenylmethane sulfonyl fluoride and a protease inhibitor cocktail for $30 \mathrm{~min}$ at $4^{\circ} \mathrm{C}$, and following centrifugation at $12,000 \mathrm{x}$ g for $15 \mathrm{~min}$ at $4^{\circ} \mathrm{C}$, the supernatants were collected. Equal amounts of extracted protein samples $(25 \mu \mathrm{g})$ were resolved by $10 \%$ SDS-PAGE and transferred 
Table I. Clinicopathological characteristics of the SLE samples and healthy controls.

\begin{tabular}{lcc}
\hline Characteristic & SLE & Healthy controls \\
\hline Number & 210 & 210 \\
Age, years & $35.8 \pm 13.5$ & $35.2 \pm 13.8$ \\
Sex & & \\
Female (\%) & $202(96.2 \%)$ & $203(96.67 \%)$ \\
Male (\%) & $8(3.8 \%)$ & $7(3.33 \%)$ \\
SLEDAI score & $8.2 \pm 5.7$ & - \\
WBCs, x10 ${ }^{9} / 1$ & $5.38 \pm 2.07$ & $5.82 \pm 2.44$ \\
Lymphocytes, $\%$ & $14.54 \pm 9.73^{\mathrm{a}}$ & $20.56 \pm 5.23$ \\
ANA+ $(\%)$ & $210(100 \%)^{\mathrm{a}}$ & 0 \\
Serum complement 3 & $0.81 \pm 0.42$ & - \\
Serum complement 4 & $0.22 \pm 0.18$ & - \\
\hline
\end{tabular}

Data are presented as the mean \pm standard deviation. ${ }^{a} \mathrm{P}<0.05$ vs. healthy control group. SLE, systemic lupus erythematosus; SLEDAI, SLE disease activity index; WBCs, white blood cells; ANA, anti-nuclear antibody.

Table II. Clinicopathological characteristics of the active SLE and stable SLE samples.

\begin{tabular}{lcc}
\hline Characteristic & Active SLE & Stable SLE \\
\hline Number & 103 & 107 \\
Age, years & $35.3 \pm 14.2$ & $34.9 \pm 14.9$ \\
Sex & & \\
Female (\%) & $100(97.09 \%)$ & $102(95.33 \%)$ \\
Male (\%) & $3(2.91 \%)$ & $5(4.67 \%)$ \\
SLEDAI score & $14.2 \pm 3.1^{\mathrm{a}}$ & $4.9 \pm 2.2$ \\
dsDNA + (\%) & $83(80.59 \%)^{\mathrm{a}}$ & $21(19.63 \%)$ \\
ANA+ (\%) & $103(100 \%)$ & $107(100 \%)$ \\
Serum complement 3 & $0.51 \pm 0.22^{\mathrm{a}}$ & $0.82 \pm 0.28$ \\
Serum complement 4 & $0.19 \pm 0.18^{\mathrm{a}}$ & $0.27 \pm 0.19$
\end{tabular}

Data are presented as the mean \pm standard deviation. ${ }^{\mathrm{a}} \mathrm{P}<0.05$ vs. stable SLE group. SLE, systemic lupus erythematosus; SLEDAI, SLE disease activity index; dsDNA, double stranded DNA; ANA, anti-nuclear antibody.

onto nitrocellulose membranes, followed by blocking with 5\% non-fat milk for $30 \mathrm{~min}$ at room temperature and incubation at $4^{\circ} \mathrm{C}$ overnight with anti-USP7, anti-IFNAR 1 and anti- $\beta$-actin primary antibodies. Membranes were then incubated with horseradish peroxidase-conjugated secondary antibodies for $2 \mathrm{~h}$ at room temperature. Protein bands were visualized using an enhanced chemiluminescence assay system (Sigma Aldrich; Merck KGaA). The experiments were repeated at least three times.

Glutathione S-transferase (GST) pull down analysis. To detect in vitro binding between USP7 and IFNAR1, the GST fusion construct GST-USP7 was purified from BL21 Escherichia coli cells (TransGen), as previously described (21). The in vitro transcription and translation of FLAG-tagged IFNAR1 was obtained from the rabbit reticulocyte lysate (TNT systems, Promega Corporation, Madison, WI, USA) and pulled down with glutathione-Sepharose beads (GE Healthcare Life Sciences, Little Chalfont, UK), according to the manufacturer's protocol.

Statistical analysis. Statistical analysis was performed using SPSS software version 17.0 (SPSS, Inc., Chicago, IL, USA). All data were presented as the mean \pm standard deviation, unless otherwise stated, of one representative of three experiments. Spearman's rank correlation was used to measure correlations between patient variables and SLE presence or activity. A paired Student's t-test was used to perform the analysis. $\mathrm{P}<0.05$ was considered to indicate a statistically significant difference.

\section{Results}

The clinical characteristics in patients with SLE and healthy controls. As demonstrated in Table I, no significant differences were observed in age or sex distribution between the SLE patients and healthy controls. The 210 SLE patients were positive for ANA, whereas the controls were negative; the proportion of lymphocytes was significantly lower in patients compared with the control, while the difference in the average number of total white blood cells was not deemed significant. As mentioned, an SLEDAI score $\geq 10$ was considered as active SLE and there were $103(49.05 \%)$ active patients and 107 (50.95\%) stable patients. As demonstrated in Table II, no significant differences were observed in age or sex distribution between these two groups. The differences in SLEDAI score, dsDNA positivity and levels of complement 3 and 4 were significant between the two groups $(\mathrm{P}<0.05)$.

Higher USP7 expression is observed in patients with SLE. The deubiquitinases take part in numerous cellular processes, but very little is known concerning the role of USPs in immune cells and specifically in T cells. In the present study, the mRNA expression level of different USP proteins between SLE and the healthy controls was screened. The expression levels of three USP proteins (USP7, USP10 and USP21) were higher in SLE patients compared with controls (data not shown). Among the USP proteins, the expression of USP7 in samples obtained from the 210 SLE patients was the most significantly upregulated compared with the normal controls (Fig. 1A). The role of USP7 in SLE was explored and analysis performed to measure whether there was any correlation between USP7 levels and the clinical features of SLE. As demonstrated, the high USP7 expression correlated positively with SLE cutaneous manifestations including the presence of skin, renal and neurological diseases (Fig. 1B). SLE patients with concurrent proteinuria had higher USP7 levels compared with those without proteinuria (Fig. 1C).

IFNARI identifies as a USP7-interacting protein. One of the biggest challenges in studying USP families is to identify their substrate and to correlate their dysregulation with pathogenesis. Affinity purification and mass spectrometry were used to detect the USP7 association proteins in vivo (data not shown). 

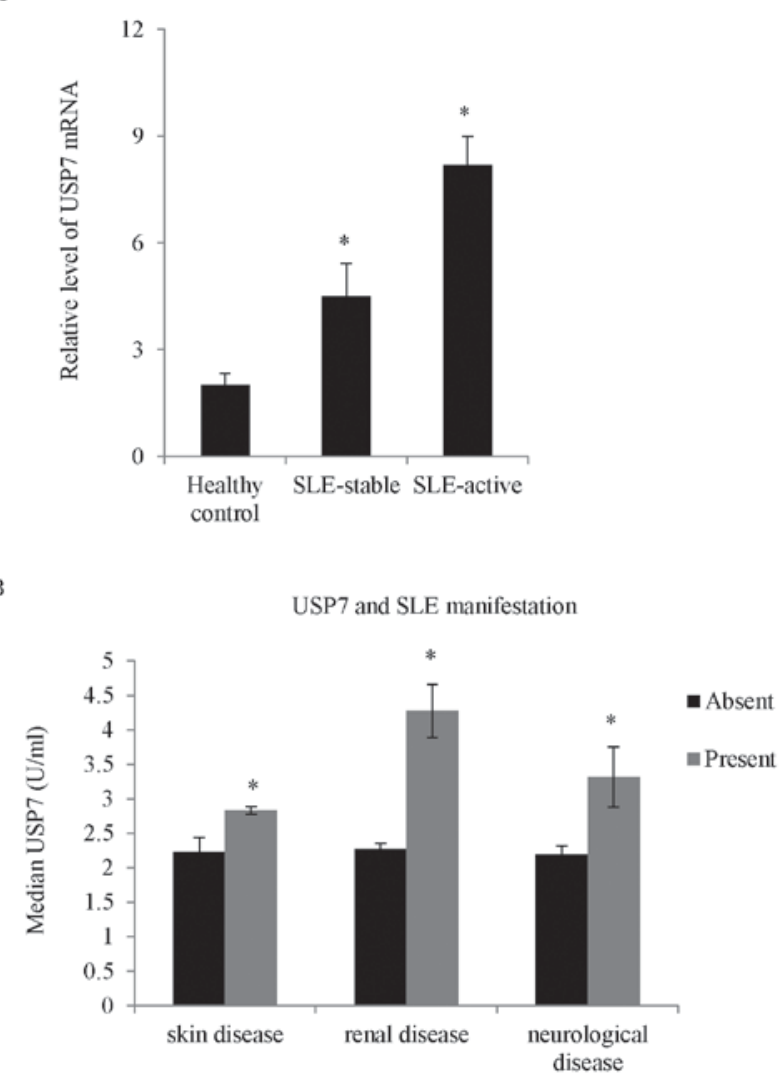

C

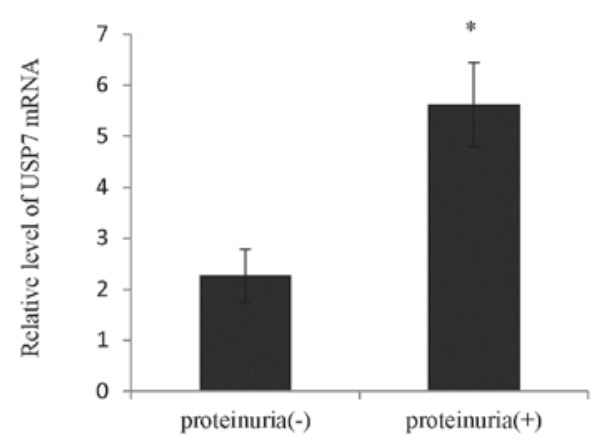

Figure 1. Higher USP7 expression was observed in patients with SLE. (A) The expression of USP7 in 210 patients with 103 active SLE, 107 stable patients with inactive SLE and 210 normal controls were measured. (B) The correlation between USP7 expression and SLE skin, renal and neurological disease. (C) Association of USP7 expression with the concurrent presence of proteinuria in SLE patients. ${ }^{*} \mathrm{P}<0.05$, as indicated. USP7, ubiquitin-specific-processing protease 7; SLE, systemic lupus erythematosus.

IFNAR1 was identified as a USP7-interacting protein. To further confirm the interaction between USP7 and IFNAR1 in vivo, a co-IP assay was performed in T cells and total protein extracted, IP with USP7 antibodies followed by immunoblotting (IB) with the antibodies against IFNAR1 indicated that USP7 co-immunoprecipitated with IFNAR1; normal rabbit IgG was used as a negative control (Fig. 2A). Reciprocally, IP was performed with anti-IFNAR1 followed by IB with anti-USP7 (Fig. 2A bottom panel). To further support the in vivo interaction between USP7 and IFNAR1, endogenous proteins from HEK-293T cells were used to confirm the interaction (Fig. 2B).
A

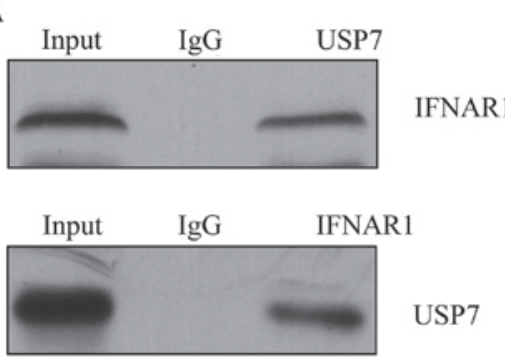

B

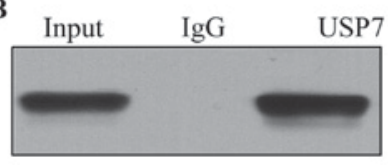

IFNAR1

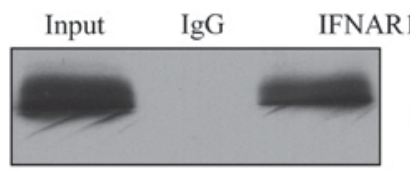

USP7

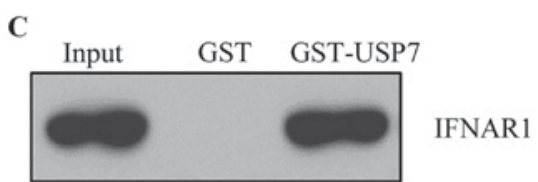

Figure 2. IFNAR1 was identified as a USP7-interacting protein. (A) Co-immunoprecipitation assay was performed. Whole $\mathrm{T}$ cell lysates were immunoprecipitated with antibodies against USP7, or normal IgG as the negative control. The immunocomplexes were then immunoblotted with IFNAR1, followed by the reverse procedure. (B) Whole HEK-293T cell lysate was used to detect the association between USP7 and IFNAR1. (C) GST pull-down assays was carried out to detect the association between USP7 and IFNAR1. The GST-fused USP7 and in vitro transcribed/translated IFNAR1 were incubated and western blotting used to detect the immunocomplexes. IFNAR1, human interferon $\alpha-2$ receptor; USP7, ubiquitin-specific-processing protease 7; Ig, immunoglobulin; GST, glutathione S-transferase.

Other USP proteins including USP10 and USP21 were additionally detected; neither had any interaction with IFNAR1, and data was not shown. To further confirm the interaction between IFNAR1 and USP7 in vitro, GST pull down assay was performed and incubation of GST-fused USP7 with in vitro transcribed/translated IFNAR1 revealed that USP7 interacted with IFNAR1 directly (Fig. 2C).

USP7 inhibits IFNAR1 ubiquitination and stabilizes IFNARI in vivo. In SLE patients, it was hypothesized that USP7 regulated IFNAR1 expression in T cells and higher USP7 expression contributed to the observed elevated IFNAR1 expression. Therefore, human primary T cells were transfected with USP7 siRNAs, control siRNAs, USP7 overexpression lentivirus or vector lentivirus. The successful knockdown efficiency (Fig. 3A) and ectopic expression of USP7 (Fig. 3B) in primary $\mathrm{T}$ cells was verified by RT-qPCR and western blot analysis, which demonstrated that the knockdown of USP7 following transfection resulted in a significant decrease in USP7 expression, while the overexpression of USP7 lentivirus resulted in a clear increase in USP7 expression.

Following USP7 knock down, the expression of IFNAR1 was measured and although the IFNAR1 mRNA level was not changed (Fig. 3C, left), the IFNAR1 protein level was markedly 

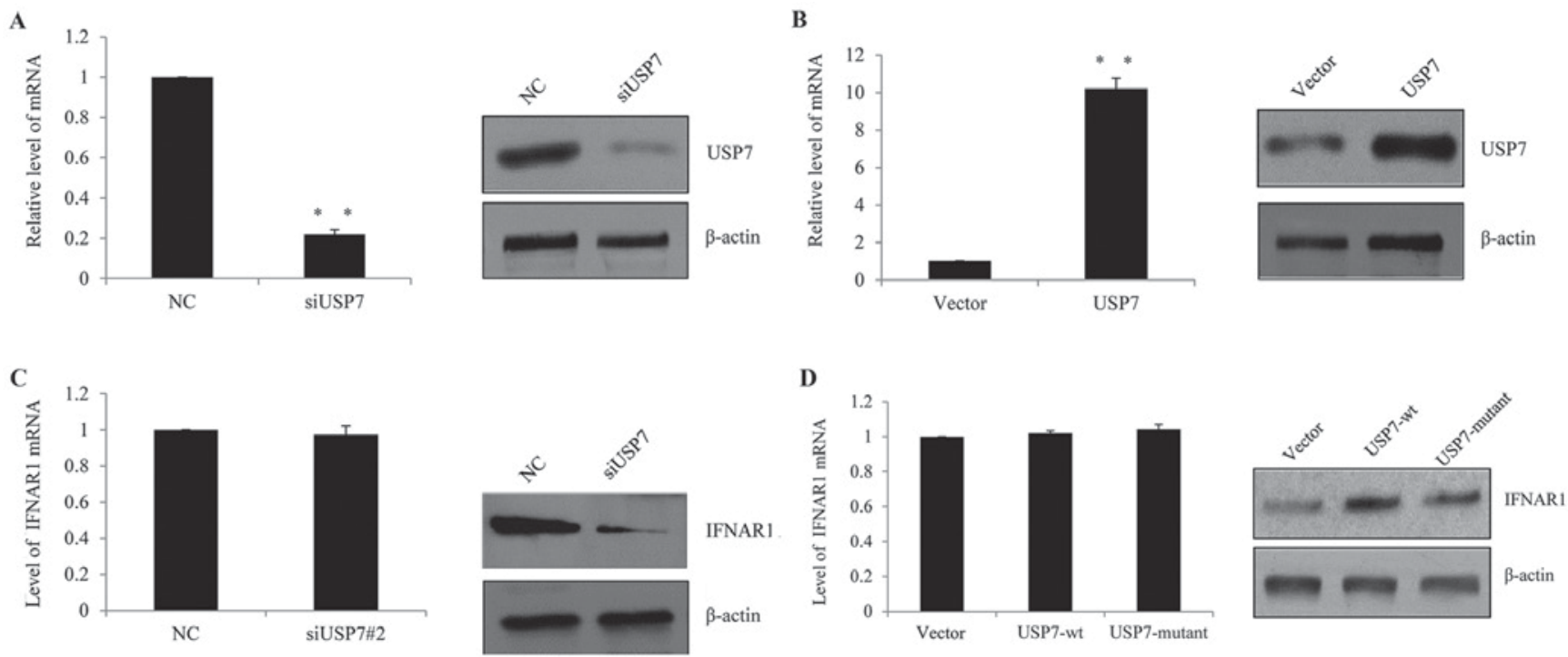

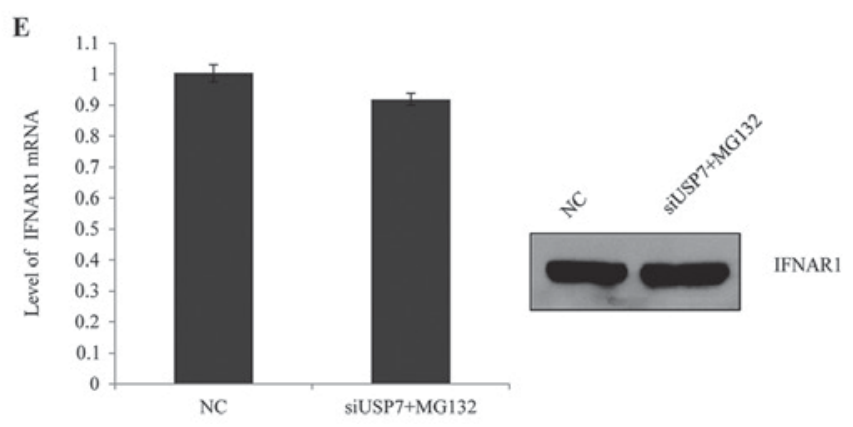

Figure 3. USP7 inhibited IFNAR1 ubiquitination and stabilized IFNAR1 in vivo. (A) The knockdown efficiency of USP7 was confirmed by RT-qPCR (left panel) and western blotting (right panel). Human primary T cells were transfected with control siRNA, siUSP7\#1 or siUSP7\#2. ${ }^{* *} \mathrm{P}<0.01$, as indicated. (B) The overexpression efficiency of USP7 was confirmed by RT-qPCR (left panel) and western blotting (right panel). Human primary T cells were transfected with vector, or USP7 overexpression lentivirus. ${ }^{* *} \mathrm{P}<0.01$, as indicated. (C) T cells were transfected with control siRNA or siUSP7 and the mRNA and protein level of IFNAR1 was detected. (D) T cells were transfected with vector, wt-USP7 or USP7 mutant lentivirus, mRNA and the protein level of IFNAR1 was detected. (E) Following USP7 knockdown in T cells, cells were incubated with the proteasome-specific inhibitor MG132 prior to harvesting, and RT-qPCR and western blot analysis were used to measure IFNAR1 expression. USP7, ubiquitin-specific-processing protease 7; IFNAR1, human interferon $\alpha$-2 receptor; RT-qPCR, reverse transcription-quantitative polymerase chain reaction; si, short interfering; wt, wild type; NC, negative control.

reduced (Fig. 3C, right). Consistently, when wild-type (wt) USP7 lentivirus or catalytically inactive USP7 (USP7/C223A) mutant lentivirus was transfected into the cells, the protein level of IFNAR1 increased in wt-USP7 transfected groups, but not in the catalytically inactive USP7 (USP7/C223A) mutant groups (Fig. 3D); the two groups demonstrated no remarkable change in the mRNA level. This indicated that USP7 may influence IFNAR1 through post-transcription modification.

To determine whether the effect of USP7 was dependent on IFNAR1 protein deubiquitination, USP7 was knocked down by siRNA and cells were harvested following MG132 (a proteasome-specific inhibitor) treatment; it was demonstrated that MG132 was successful in saving IFNAR1 protein from degradation in USP7 knockdown groups compared with the control groups (Fig. 3E). Based on the above, it was hypothesized that USP7 stabilized IFNAR1, as it protected the protein from ubiquitination.

Effects of USP7 on activation of the IFNa pathway. Whether sustained overexpression of USP7 was able to induce the activation of the type I IFN pathway in SLE patients was explored.
The activation of STAT proteins was a response to type I IFN, since the IFN-stimulated transcription factor 3 complex contained three core subunits that activated STAT-1 and STAT-2 in addition to interferon regulatory factor (IRF)-9, the function of which is to initiate transcription of IFN-inducible genes. It was next considered whether USP7 regulated downstream target transactivation following stimulation with type I IFN. As demonstrated from mRNA and protein levels in Fig. 4A and B, normal PBMCs were transfected and stimulated with type I IFN, and knockdown of USP7 consistently reduced the expression of STAT-1 and STAT-2.

Furthermore, knockdown of USP7 notably reduced the mRNA (Fig. 4C) and protein expression levels (Fig. 4D) of selected IFN-inducible genes, including IFN-induced protein with tetratricopeptide repeats 3 (IFIT3), myxovirus resistance 1 (MX1) and 2',5'-oligoadenylate synthetase 1 (OAS1).

Correlation between USP7 expression and SLE disease activity. It has been reported by several groups $(22,5)$ that type I IFN exhibits a key etiological role in SLE since, by stabilizing IFNAR1, USP7 may reflect defects in positive regulation of the 
A

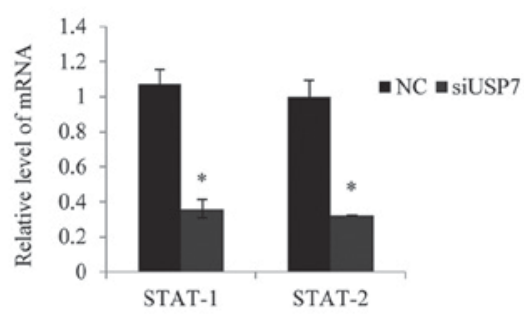

B

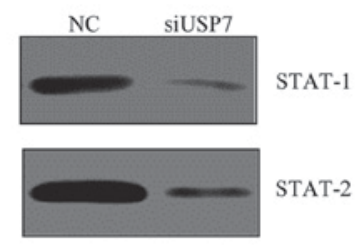

C

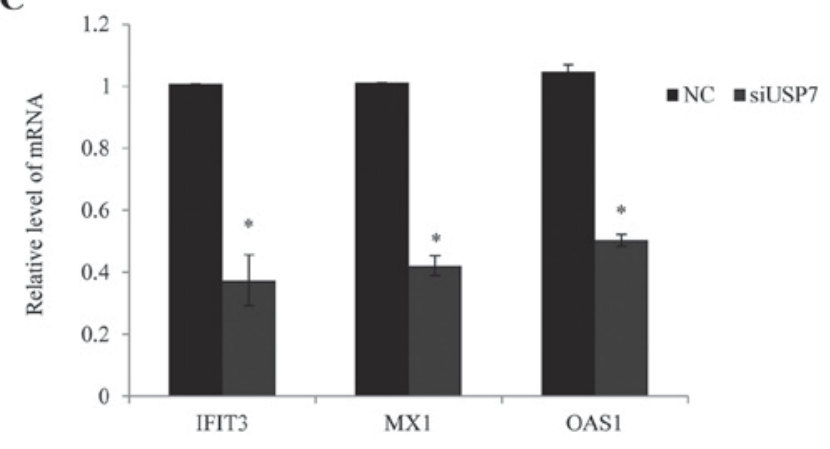

D

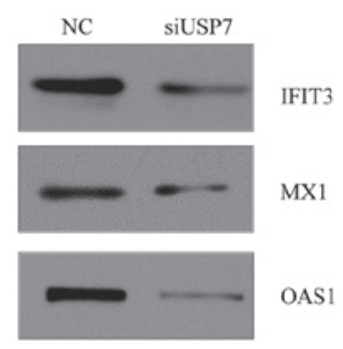

Figure 4. Effects of USP7 on the activation of IFN $\alpha$ pathway. (A) PBMCs were transfected with control siRNA and siUSP7, followed by incubation with type I IFN for $6 \mathrm{~h}$. The mRNA levels of STAT-1 and STAT-2 were measured, GAPDH was used as an internal normalization control. Error bars represent standard error of the mean. ${ }^{\mathrm{P}}<0.05$, as indicated. (B) Following the above treatment, the protein levels of STAT-1, STAT-2 and IRF-9 were measured by western blotting and $\beta$-actin was used as an internal control Following the above treatment, the (C) mRNA level and (D) protein level of three IFN-inducible genes, IFIT3, MX1 and OAS1 were quantified. Values are the mean + standard error. USP7, ubiquitin-specific-processing protease 7; IFN, interferon; PBMCs, peripheral blood mononuclear cells; si, short interfering; STAT, signal transducer and activator of transcription; IRF, interferon regulatory factor; IFIT3, IFN-induced protein with tetratricopeptide repeats 3; MX1, myxovirus resistance 1; OAS1, 2',5'-oligoadenylate synthetase $1 ; \mathrm{NC}$, negative control.

immune response. The associations were therefore analyzed and, as expected, a positive correlation was identified between USP7 levels and IFN scores (Fig. 5A).

A direct positive correlation was observed between USP7 levels and the SLEDAI scores (Fig. 5B), and between the USP7 levels and the anti-dsDNA levels (Fig. 5C). Furthermore, the IFN level also correlated positively with SLEDAI scores and
A

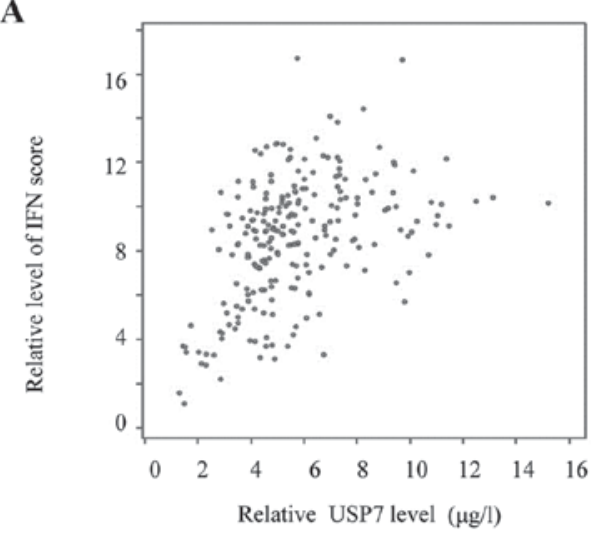

B

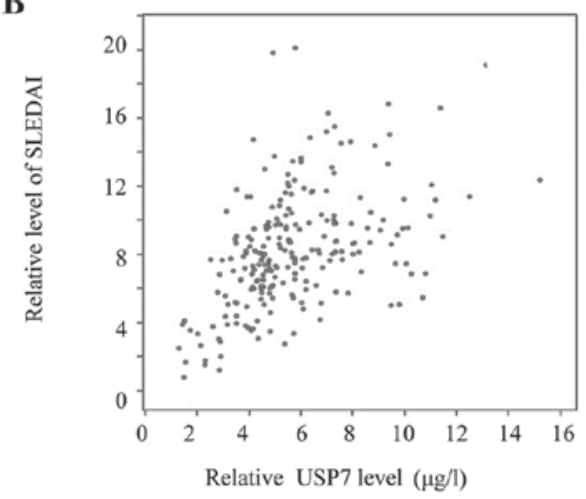

C

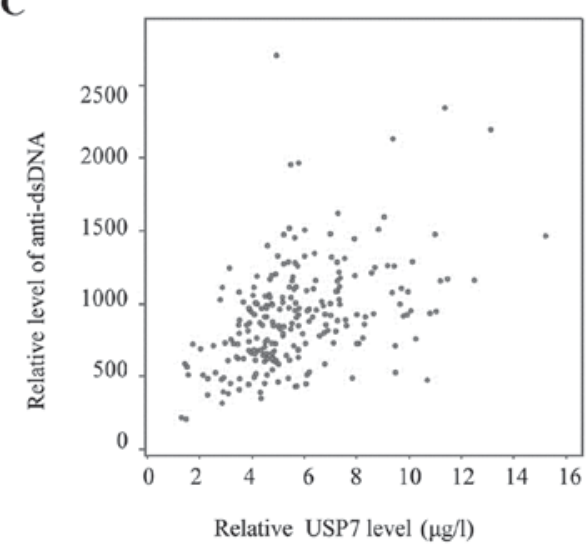

Figure 5. Correlation between USP7 expression and SLE disease activity. Correlation was measured between the expression of (A) USP7 in SLE patients and activation of the type I interferon pathway; (B) USP7 in SLE patients and SLEDAI scores; and, (C) USP7 in SLE patients and the anti-dsDNA scores. USP7, ubiquitin-specific-processing protease 7; SLE, systemic lupus erythematosus; DAI, disease activity index; ds, double stranded.

the anti-dsDNA levels (data not shown); the study conducted by Dall'era et al (23) reported a similar tendency. It was concluded that USP7 expression levels correlate positively with SLE disease activity via stabilization of IFNAR1.

\section{Discussion}

Increasing evidence has indicated that IFN $\alpha$ is associated with the progression of several diseases and may serve as a target for therapy. The role of the USP family in autoimmunity is only just beginning to be explored. 
The results of the present study established that USP7 is upregulated in patients with SLE, and that greater expression of USP7 was associated with skin, renal and neurological diseases. SLE patients with concurrent proteinuria had higher USP7 levels than those without, which indicated that greater USP7 levels may result in organ damage.

Furthermore, investigation provided insight into the mechanism of the regulation by USP7; bioinformatics tools were used to search for its potential association with the key components involved in SLE (5), and this led to the identification of IFNAR1 as a potential USP7 interaction protein. As it was hypothesized that USP7 interacted with IFNAR1 in vivo and in vitro, co-IP and GST-pull down assays were used to confirm that USP7 disassembled IFNAR1-dependent poly-ubiquitin chains and stabilized IFNAR1 in vivo. As the type I IFN pathway has been reported as a significant contributor to the pathogenesis of SLE (5), the activation effects of USP7 on the IFN $\alpha$ pathway were investigated. When USP7 was knocked down, the signaling downstream of IFN, including the expression of STAT-1, STAT-2 and its predicted IFN-inducible genes (IFIT3, MX1 and OAS1), were downregulated. For the first time, to the best of the authors' knowledge, it has been demonstrated that USP7 is significantly overexpressed in SLE patients compared with healthy controls. Furthermore, a positive correlation was observed between USP7 levels, IFN scores, SLEDAI scores and anti-dsDNA, which indicated that the activation of the type I IFN pathway in SLE patients is due to USP7 over-activation in the pathogenesis of autoimmune conditions. In conclusion, the results of the present study demonstrated that the overexpression of USP7 is relevant to the biologic and clinical behavior of SLE. The findings suggested that the USP family may serve as therapeutic targets via regulation of IFNAR1 for the treatment of SLE. In the future, knockout and transgenic animal models will be required to further identify the role of USP7 in autoimmune diseases.

\section{References}

1. Tsokos GC: Systemic lupus erythematosus. N Engl J Med 365 2110-2121, 2011.

2. Gillis JZ, Panopalis P, Schmajuk G, Ramsey-Goldman R and Yazdany J: Systematic review of the literature informing the systemic lupus erythematosus indicators project: Reproductive health care quality indicators. Arthritis Care Res (Hoboken) 63: 17-30, 2011.

3. Hedrich CM and Tsokos GC: Epigenetic mechanisms in systemic lupus erythematosus and other autoimmune diseases. Trends Mol Med 17: 714-724, 2011.

4. Qu B and Shen N: miRNAs in the pathogenesis of systemic lupus erythematosus. Int J Mol Sci 16: 9557-9572, 2015.

5. Crow MK: Advances in understanding the role of type I interferons in systemic lupus erythematosus. Curr Opin Rheumatol 26 467-474, 2014.
6. Thacker SG, Zhao W, Smith CK, Luo W, Wang H, Vivekanandan-Giri A, Rabquer BJ, Koch AE, Pennathur S, Davidson A, et al: Type I interferons modulate vascular function, repair, thrombosis, and plaque progression in murine models of lupus and atherosclerosis. Arthritis Rheum 64: 2975-2985, 2012.

7. Lee PY, Li Y, Richards HB, Chan FS, Zhuang H, Narain S, Butfiloski EJ, Sobel ES, Reeves WH and Segal MS: Type I interferon as a novel risk factor for endothelial progenitor cell depletion and endothelial dysfunction in systemic lupus erythematosus. Arthritis Rheum 56: 3759-3769, 2007.

8. Denny MF, Thacker S, Mehta H, Somers EC, Dodick T, Barrat FJ, McCune WJ and Kaplan MJ: Interferon-alpha promotes abnormal vasculogenesis in lupus: A potential pathway for premature atherosclerosis. Blood 110: 2907-2915, 2007.

9. Reynier F, Petit F, Paye M, Turrel-Davin F, Imbert PE, Hot A, Mougin B and Miossec P: Importance of correlation between gene expression levels: Application to the type I interferon signature in rheumatoid arthritis. PLoS One 6: e24828, 2011.

10. Müller U, Steinhoff U, Reis LF, Hemmi S, Pavlovic J, Zinkernagel RM and Aguet M: Functional role of type I and type II interferons in antiviral defense. Science 264: 1918-1921, 1994.

11. Kumar KG, Tang W, Ravindranath AK, Clark WA, Croze E and Fuchs SY: SCF(HOS) ubiquitin ligase mediates the ligand-induced down-regulation of the interferon-alpha receptor. EMBO J 22: 5480-5490, 2003.

12. Everett RD, Meredith M, Orr A, Cross A, Kathoria M and Parkinson J: A novel ubiquitin-specific protease is dynamically associated with the PML nuclear domain and binds to a herpesvirus regulatory protein. EMBO J 16: 1519-1530, 1997.

13. Zhou Z, Yao X, Li S, Xiong Y, Dong X, Zhao Y, Jiang J and Zhang Q: Deubiquitination of Ci/Gli by Usp7/HAUSP regulates hedgehog signaling. Dev Cell 34: 58-72, 2015.

14. Song MS, Salmena L, Carracedo A, Egia A, Lo-Coco F, Teruya-Feldstein J and Pandolfi PP: The deubiquitinylation and localization of PTEN are regulated by a HAUSP-PML network. Nature 455: 813-817, 2008.

15. van der Horst A, de Vries-Smits AM, Brenkman AB, van Triest MH, van den Broek N, Colland F, Maurice MM and Burgering BM: FOXO4 transcriptional activity is regulated by monoubiquitination and USP7/HAUSP. Nat Cell Biol 8: 1064-1073, 2006.

16. van der Knaap JA, Kumar BR, Moshkin YM, Langenberg K, Krijgsveld J, Heck AJ, Karch F and Verrijzer CP: GMP synthetase stimulates histone $\mathrm{H} 2 \mathrm{~B}$ deubiquitylation by the epigenetic silencer USP7. Mol Cell 17: 695-707, 2005.

17. Li M, Chen D, Shiloh A, Luo J, Nikolaev AY, Qin J and Gu W: Deubiquitination of p53 by HAUSP is an important pathway for p53 stabilization. Nature 416: 648-653, 2002.

18. Hochberg MC: Updating the American College of Rheumatology revised criteria for the classification of systemic lupus erythematosus. Arthritis Rheum 40: 1725, 1997.

19. Gladman DD, Ibañez D and Urowitz MB: Systemic lupus erythematosus disease activity index 2000. J Rheumatol 29: 288-291, 2002.

20. Livak KJ and Schmittgen TD: Analysis of relative gene expression data using real-time quantitative PCR and the 2(-Delta Delta $\mathrm{C}(\mathrm{T})$ ) method. Methods 25: 402-408, 2001.

21. Einarson MB, Pugacheva EN and Orlinick JR: GST Pull-down. CSH Protoc 2007: pdb prot4757, 2007.

22. Zhang ZM, Rothbart SB, Allison DF, Cai Q, Harrison JS, Li L, Wang Y, Strahl BD, Wang GG and Song J: An allosteric interaction links USP7 to deubiquitination and chromatin targeting of UHRF1. Cell Rep 12: 1400-1406, 2015.

23. Dall'era MC, Cardarelli PM, Preston BT, Witte A and Davis JC Jr: Type I interferon correlates with serological and clinical manifestations of SLE. Ann Rheum Dis 64: 1692-1697, 2005. 